\title{
Enhanced Resolution from Full-Field Ptychography with an Electron Microscope Pixel Array Detector
}

\author{
Yi Jiang ${ }^{1}$, Yimo $\operatorname{Han}^{2}$, Zhen Chen ${ }^{2}$, Veit Elser ${ }^{1}$, David A. Muller ${ }^{2,3}$ \\ 1. Department of Physics, Cornell University, Ithaca NY, USA \\ 2. School of Applied and Engineering Physics, Cornell University, Ithaca NY, USA \\ 3. Kavli Institute at Cornell for Nanoscale Science, Ithaca NY, USA
}

In STEM, complete information about the scattering potential of a sample is encoded in the distribution of transmitted electrons. To measure the entire diffraction pattern, a new generation of high-speed, momentum-resolved detectors has been developed, enabling new imaging modes. Unfortunately, most of the new detectors were designed for low-dose x-ray and biological imaging. Their pulse counting architectures are limited to beam currents of less than $\sim 0.1 \mathrm{pA} /$ pixel, making them poorly suited to highspeed scanning electron diffraction applications.

Recently, we developed electron microscope pixel array detector (EMPAD) [1] that has a high dynamic range $(1,000,000: 1)$ while preserving single electron sensitivity. The camera has fast readout speed $(0.86$ $\mathrm{ms} /$ frame) and can record all the scattered electrons for atomic-resolution probes. These properties open the doors for new quantitative imaging applications including strain fields in 2D materials [2] and polarization vortices in ferroelectrics [3].

Here we show that applying electron ptychography to full diffraction patterns obtained by EMPAD leads to higher resolution reconstructions than ADF-STEM or prior bright-field ptychography techniques. Figure 1(a) shows a diffraction pattern extracted from a single molybdenum atom from a 4D data set of a monolayer $\mathrm{MoS}_{2}$ recorded on an aberration-corrected FEI Titan at $80 \mathrm{keV}$ and $7 \mathrm{pA}$. We used the super-resolution ePIE algorithm [4] and simultaneously reconstructed the transmission function and probe functions with a pixel size of 0.11 angstrom, roughly half of the scan step size. Compared with annular dark field (Figure 1b) and integrated center-of-mass images (Figure 1c), the ptychography reconstruction (Figure 1d) achieves a higher resolution and is more robust to noise and aberrations in the probe. The sulfur monovacancy (indicated by red arrows) is also well-resolved, as shown in Figure 3c.

We explored the role of higher angle diffraction information by applying a mask to each CBED pattern before ptychographic reconstruction. The cutoffs are chosen to be integer multiples of the convergence semi-angle $(\alpha=21.4 \mathrm{mrad})$. When only using the central bright-field disk, the reconstructed phase (Figure 2a) is similar in resolution to iCoM and ADF. As the cutoff increases, atoms become sharper until about $3 \alpha$ (Figure 2b-d). However, the probe's shape is similar at different cutoffs (Figure 3a), indicating resolution improvement is mainly due to the electrons collected at high scattering angles.

\section{References:}

[1] M. Tate et al, Microscopy and Microanalysis 22 (2016), p. 237-249.

[2] Y. Han et al, Microscopy and Microanalysis 22, (2016), p. 870-871.

[3] K. Nguyen et al, Microscopy and Microanalysis 22, (2016), p. 472-473.

[4] A. Maiden et al, J. Opt. Soc. Am. 28 (2011), p.604-612.

[5] Y. Jiang is supported by DOE grant DE-SC0005827. Facility support from NSF DMR-1120296. Helpful discussion with Martin Humphries and Peter Nellist are acknowledged. 

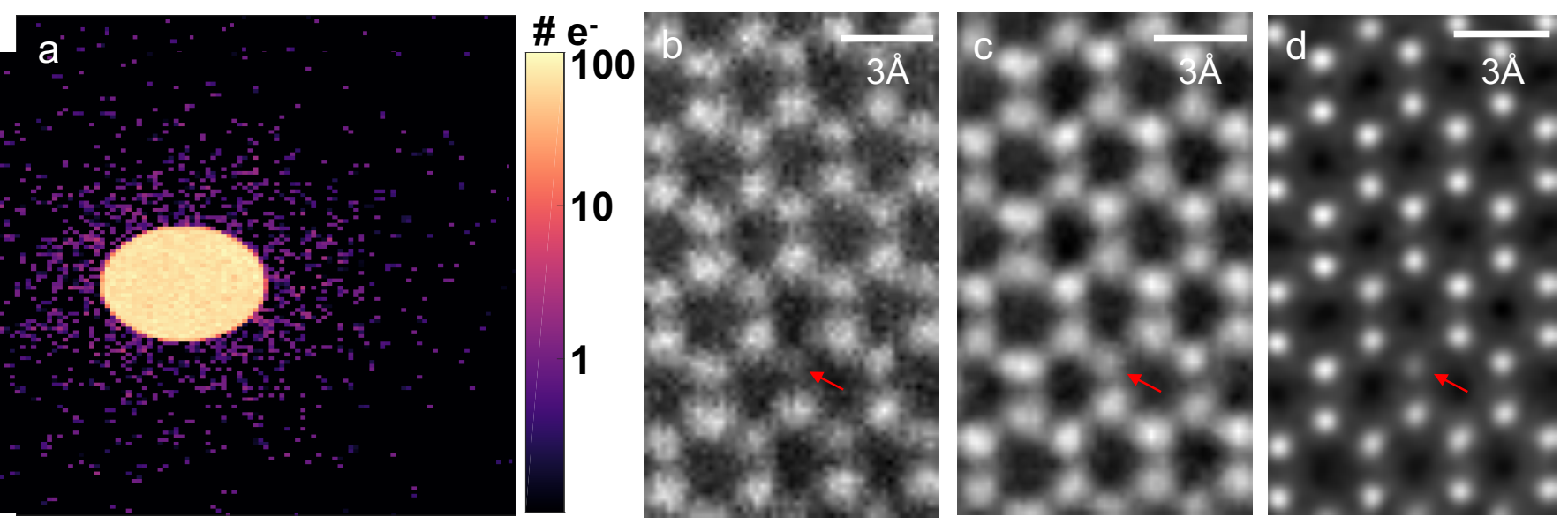

Figure 1. (a) CBED pattern from a monolayer $\mathrm{MoS}_{2}$. (b) ADF image. (c) Integrated center-of-mass image. (d) Phase of the specimen transmission function reconstructed by ptychography.
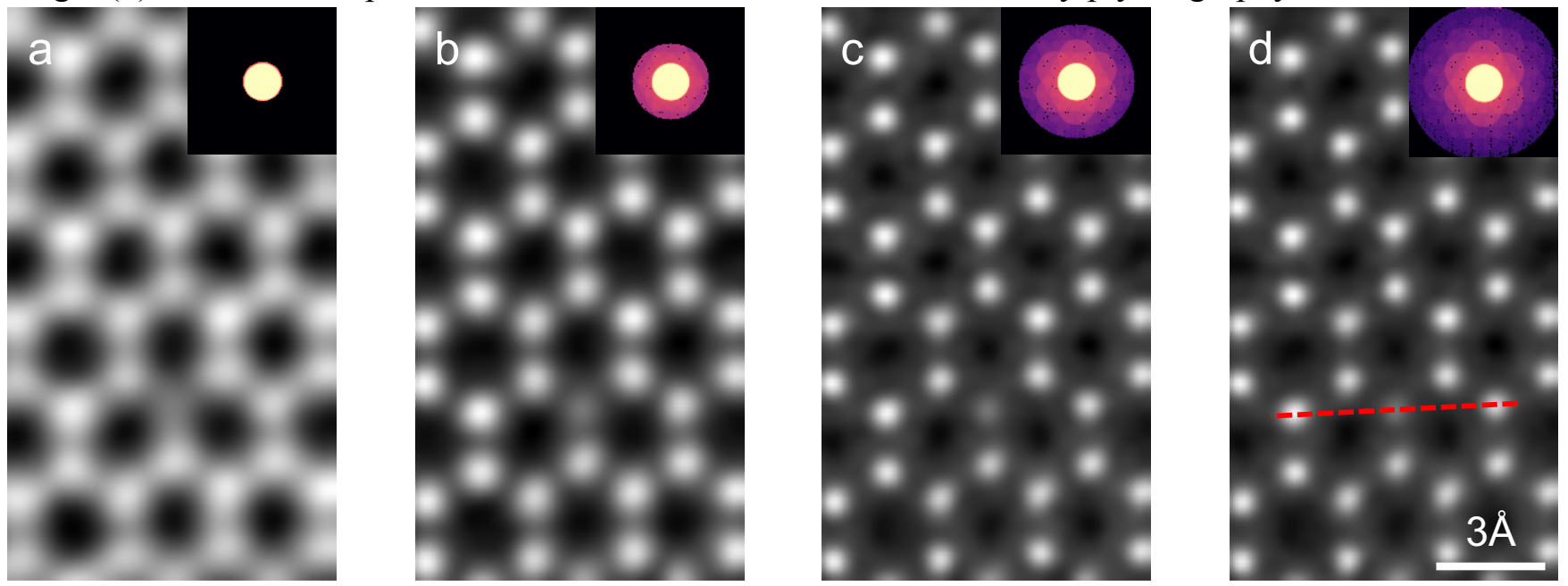

Figure 2. Ptychography reconstructions using data collected within cutoff angles of $\alpha, 2 \alpha, 3 \alpha$ and $4 \alpha$, where $\alpha$ is the convergence semi-angle. The average CBED patterns are shown at the top-right corners.
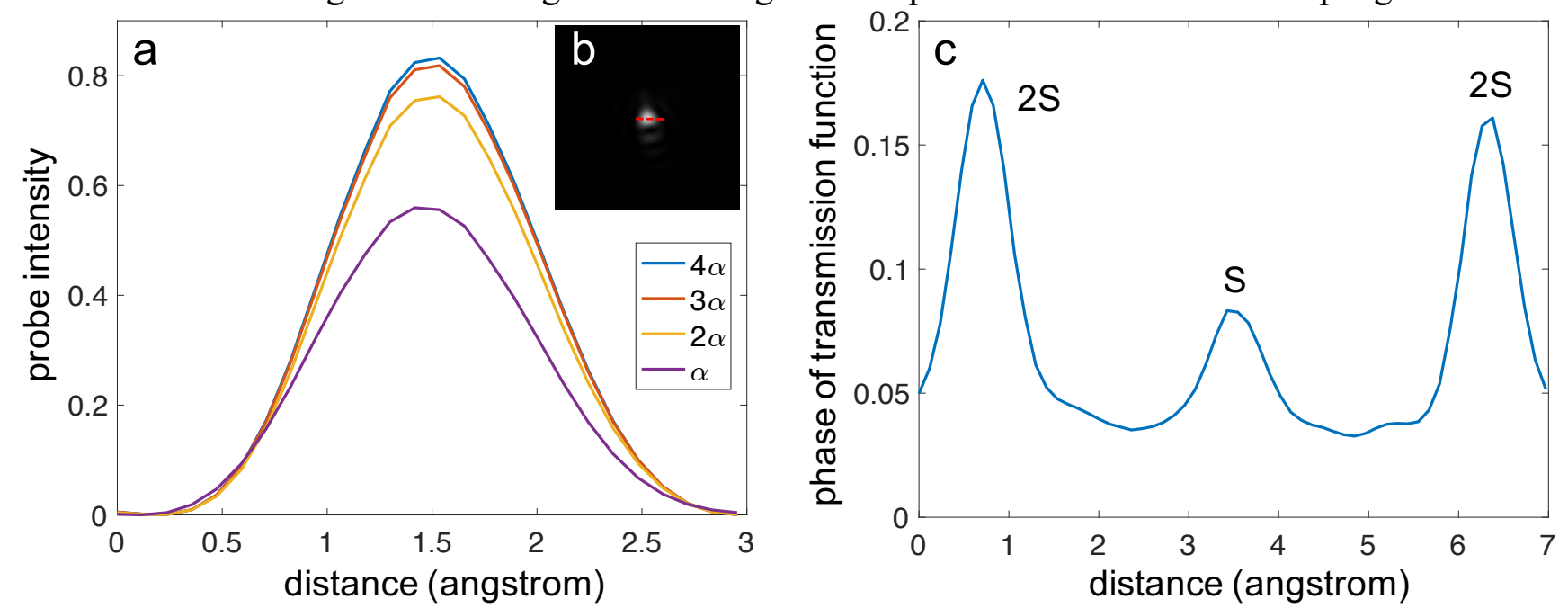

Figure 3. (a) Line profiles across probe intensity reconstructed using data collected within different cutoff angles. (b) Reconstructed probe intensity at $4 \alpha$. (c) Line profile across three sulfur (S) columns as indicated in Figure 2d. Tails from the Mo atoms are present at 2 and $5 \AA$. 